This is the author's final, peer-reviewed manuscript as accepted for publication. The publisher-formatted version may be available through the publisher's web site or your institution's library.

\title{
Robust variable selection through MAVE
}

Weixin Yao, Qin Wang

\section{How to cite this manuscript}

If you make reference to this version of the manuscript, use the following information:

Yao, W., \& Wang, Q. (2013). Robust variable selection through MAVE. Retrieved from http://krex.ksu.edu

\section{Published Version Information}

Citation: Yao, W., \& Wang, Q. (2013). Robust variable selection through MAVE. Computational Statistics and Data Analysis, 63, 42-49.

Copyright: ( 2013 Elsevier B.V.

Digital Object Identifier (DOI): doi:10.1016/j.csda.2013.01.021

Publisher's Link: http://www.sciencedirect.com/science/article/pii/S0167947313000364

This item was retrieved from the K-State Research Exchange (K-REx), the institutional repository of Kansas State University. K-REx is available at http://krex.ksu.edu 


\title{
Robust Variable Selection Through MAVE
}

\author{
Weixin $\mathrm{Yao}^{\mathrm{a}}$, Qin Wang*,b \\ ${ }^{a}$ Department of Statistics, Kansas State University, \\ Manhattan, Kansas 66506, U.S.A. \\ ${ }^{b}$ Department of Statistical Sciences and Operations Research, \\ Virginia Commonwealth University, Richmond, Virginia 23284, U.S.A.
}

\begin{abstract}
Dimension reduction and variable selection play important roles in high dimensional data analysis. The sparse MAVE, a model-free variable selection method, is a nice combination of shrinkage estimation, Lasso, and an effective dimension reduction method, MAVE (minimum average variance estimation). However, it is not robust to outliers in the dependent variable because of the use of least-squares criterion. A robust variable selection method based on sparse MAVE is developed, together with an efficient estimation algorithm to enhance its practical applicability. In addition, a robust cross-validation is also proposed to select the structural dimension. The effectiveness of the new approach is verified through simulation studies and a real data analysis.
\end{abstract}

Key words: Sufficient dimension reduction, MAVE, Shrinkage estimation, Robust estimation.

\section{Introduction}

The explosion of massive data in the last decades has generated considerable challenges and interests in the development of statistical modeling. Practically, only part of these observed variables are believed to be truly relevant to the response. Thus, variable selection plays an important role in analyzing these high dimensional data, not only for better model interpretation but also for higher prediction accuracy (Fan and Li, 2006). A lot of research

\footnotetext{
${ }^{*}$ Corresponding author.
}

Email addresses: wxyao@ksu.edu (Weixin Yao), qwang3@vcu.edu (Qin Wang)

Preprint submitted to Computational Statistics and Data Analysis

January 21, 2013 
efforts have been devoted to this area. Many traditional model-based variable selection criteria have been advocated and strengthened in the literature, such as $C_{p}$, AIC, BIC, etc. Recently a family of regularization approaches, including Nonnegative Garrote (Brieman, 1995), Lasso (Tibshirani, 1996), SCAD (Fan and Li, 2001), Lars (Efron, Hastie, and Tibshirani, 2004) and Elastic Net (Zou and Hastie, 2005), was proposed to automatically select informative variables through continuous shrinkage. However, because of the so-called 'curse of dimensionality' (Bellman, 1961), it is very difficult or even infeasible to formulate and validate a parametric model with a large number of covariates. So it is desirable to have a set of model-free variable selection approaches.

Sufficient dimension reduction ( $\mathrm{Li}, 1991$; Cook, 1998) provides such a model-free alternative to variable selection. The basic idea of sufficient dimension reduction is to replace the original high dimensional predictor vector with its appropriate low dimensional projection while preserving full regression information. Each direction in the low dimensional subspace is a linear combination of original predictors. Cook (2004) and Li, Cook, and Nachtsheim (2005) proposed several testing procedures to evaluate the contribution of each covariate. Similar to the model-based subset selection procedures, these methods are not stable because of their inherent discreteness (Brieman, 1996). Ni, Cook, and Tsai (2005), Li and Nachtsheim (2006), Li (2007), Zhou and He (2008) and Bondell and Li (2009) used regularization paradigm to incorporate shrinkage estimation into inverse regression dimension reduction methods. Along the same line, Wang and Yin (2008) combined shrinkage estimation and a forward regression dimension reduction method, MAVE (minimum average variance estimation, Xia et al. 2002), and proposed sparse MAVE to select informative covariates. Compared to the previous work, sparse MAVE is model-free and requires no strong probabilistic assumptions on the predictors. However, MAVE and sparse MAVE are not robust to outliers in the dependent variable because of the use of least-squares criterion. Čížek and Härdle (2006) gave a comprehensive study of the sensitivity of MAVE to outliers and proposed a robust enhancement to MAVE by replacing the local least squares with local L- or M- estimation.

In this article, we extend the robust estimation to variable selection and propose a robust 
sparse MAVE. It can exhaustively estimate directions in the regression mean function and select informative covariates simultaneously, while being robust to the existence of possible outliers in the dependent variable. In addition, a robust cross-validation is also proposed to select the structural dimension. The effectiveness of the new approach is verified through simulation studies and a real data analysis.

The rest of the article is organized as follows. In Section 2, we briefly review the methods MAVE and sparse MAVE. The robust extension of sparse MAVE is detailed in Section 3. Simulation studies and comparison with some existing methods are presented in Section 4. In Section 5, we apply the proposed robust sparse MAVE to a logo design data collected by Henderson and Cote (1998). Finally, in Section 6, we conclude the article with a short discussion.

\section{A brief review of MAVE and sparse MAVE}

The regression-type model of a response $y \in \mathcal{R}^{1}$ on a vector $\mathbf{x} \in \mathcal{R}^{p}$ can be written as

$$
y=g\left(\mathbf{B}^{T} \mathbf{x}\right)+\varepsilon
$$

where $g(\cdot)$ is an unknown smooth link function, $\mathbf{B}=\left(\boldsymbol{\beta}_{1}, \ldots, \boldsymbol{\beta}_{d}\right)$ is a $p \times d$ orthogonal matrix $\left(\mathbf{B}^{T} \mathbf{B}=I_{d}\right)$ with $d<p$ and $\mathrm{E}(\varepsilon \mid \mathbf{x})=0$ almost surely. Xia et al. (2002) defined the $d$ dimensional subspace $\mathbf{B}^{T} \mathbf{x}$ the effective dimension reduction (EDR) space, which captures all the information of $E(y \mid \mathbf{x})$. The $d$ is usually called the structural dimension of the EDR space. Given a random sample $\left\{\left(\mathbf{x}_{i}, y_{i}\right), i=1, \ldots, n\right\}$, the MAVE estimates the EDR directions by solving the following minimization problem

$$
\min _{\mathbf{B}, a_{j}, \mathbf{b}_{j}, j=1, \ldots, n}\left(\sum_{j=1}^{n} \sum_{i=1}^{n}\left[y_{i}-\left\{a_{j}+\mathbf{b}_{j}^{T} \mathbf{B}^{T}\left(\mathbf{x}_{i}-\mathbf{x}_{j}\right)\right\}\right]^{2} w_{i j}\right),
$$

where $\mathbf{B}^{T} \mathbf{B}=I_{d}$ and the weight $w_{i j}$ is a function of the distance between $\mathbf{x}_{i}$ and $\mathbf{x}_{j}$. The minimization of (2) can be solved iteratively with respect to $\left\{\left(a_{j}, \mathbf{b}_{j}\right), j=1, \cdots, n\right\}$ and $\mathbf{B}$ separately. The estimation of MAVE is very efficient since only two quadratic programming 
problems are involved and both have explicit solutions. To improve the estimation accuracy, a lower dimensional kernel weight $\tilde{w}_{i j}$ as a function of $\tilde{\mathbf{B}}^{T}\left(\mathbf{x}_{i}-\mathbf{x}_{j}\right)$ can be used after an initial estimate $\tilde{\mathbf{B}}$ was obtained (the refined MAVE).

Note that each reduced variable in $\mathbf{B}^{T} \mathbf{x}$ is a linear combination of all original predictors. But it is not uncommon in practice that some covariates are irrelevant among a large number of candidates. To effectively select those informative variables can improve both the model interpretability and the prediction accuracy, Wang and Yin (2008) proposed sparse MAVE to incorporate an $L_{1}$ penalty into the above estimation. The constrained optimization is as follows,

$$
\min _{\mathbf{B}, a_{j}, \mathbf{b}_{j}, j=1, \ldots, n}\left(\sum_{j=1}^{n} \sum_{i=1}^{n}\left[y_{i}-\left\{a_{j}+\mathbf{b}_{j}^{T} \mathbf{B}^{T}\left(\mathbf{x}_{i}-\mathbf{x}_{j}\right)\right\}\right]^{2} w_{i j}+\sum_{k=1}^{d} \lambda_{k}\left|\boldsymbol{\beta}_{k}\right|_{1}\right),
$$

where $|\cdot|_{1}$ represents the $L_{1}$ norm and $\left\{\lambda_{k}, k=1, \cdots, d\right\}$ are nonnegative regularization parameters which control the amount of shrinkage. Through penalizing on the $L_{1}$ norm of the parameter estimates, we can achieve the goal of variable selection when the true direction has a sparse representation. The minimization of (3) can be solved by a standard Lasso algorithm. More details can be found in Wang and Yin (2008).

\section{Robust sparse MAVE}

\subsection{Robust estimation}

Note that in (2) and (3), the least-squares criterion is used between the response and the regression function to evaluate how well the model fits. It corresponds to the maximum likelihood estimation (MLE) when the error is normally distributed. However, it is not robust to outliers in the dependent variable $y$ and to the violation of distribution assumptions on $\varepsilon$, such as heavy-tailed errors. To achieve the robustness in estimation, Čížek and Härdle (2006) proposed to replace the local least squares with local L- or M- estimation. The robust 
MAVE estimates the EDR directions by minimizing

$$
\min _{\mathbf{B}, a_{j}, \mathbf{b}_{j}, j=1, \ldots, n} \sum_{j=1}^{n} \sum_{i=1}^{n} \rho\left(y_{i}-\left\{a_{j}+\mathbf{b}_{j}^{T} \mathbf{B}^{T}\left(\mathbf{x}_{i}-\mathbf{x}_{j}\right)\right\}\right) w_{i j}
$$

where $\rho(\cdot)$ is a robust loss function. Note that the traditional least squares criterion corresponds to $\rho(t)=t^{2}$, and the median regression uses $L_{1}$ loss where $\rho(t)=|t|_{1}$. Its derivative $\psi(\cdot)=\rho^{\prime}(\cdot)$ is proportional to the influence function.

The Huber function (Huber 1981) is one commonly used robust loss function, where $\psi_{c}(t)=\rho^{\prime}(t)=\max \{-c, \min (c, t)\}$ and the tuning constant $c$ regulates the amount of robustness. Huber (1981) recommends using $c=1.345 \sigma$ in practice, where $\sigma$ is the standard deviation of $\varepsilon$. This choice produces a relative efficiency of approximately $95 \%$ when the error density is normal. Another possibility for $\psi(\cdot)$ is Tukey's bisquare function $\psi_{c}(t)=$ $t\left\{1-(t / c)^{2}\right\}_{+}^{2}$, which weighs the tail contribution of $t$ by a biweight function. In the parametric robustness literature, the use of $c=4.685 \sigma$, which produces $95 \%$ efficiency, is recommended. Figure 1 shows the comparison among these loss functions and their corresponding influence functions. More details can be found in Huber (1981), Hampel et al. (1986), Rousseeuw et al. (2003), and Maronna et al. (2006).

Note that the monotone regression M-estimators, such as the one based on Huber's function, are not robust to the high leverage outliers. However, the MAVE estimation is based on the local linear regression technique and the high leverage outliers is less likely to appear in a local window determined by the bandwidth and kernel function.

\subsection{Robust sparse $M A V E$}

To select the informative covariates robustly, an $L_{1}$ penalty can be introduced into the expression (4),

$$
\min _{\mathbf{B}, a_{j}, \mathbf{b}_{j}, j=1, \ldots, n}\left(\sum_{j=1}^{n} \sum_{i=1}^{n} \rho\left(y_{i}-\left\{a_{j}+\mathbf{b}_{j}^{T} \mathbf{B}^{T}\left(\mathbf{x}_{i}-\mathbf{x}_{j}\right)\right\}\right) w_{i j}+\sum_{k=1}^{d} \lambda_{k}\left|\boldsymbol{\beta}_{k}\right|_{1}\right)
$$



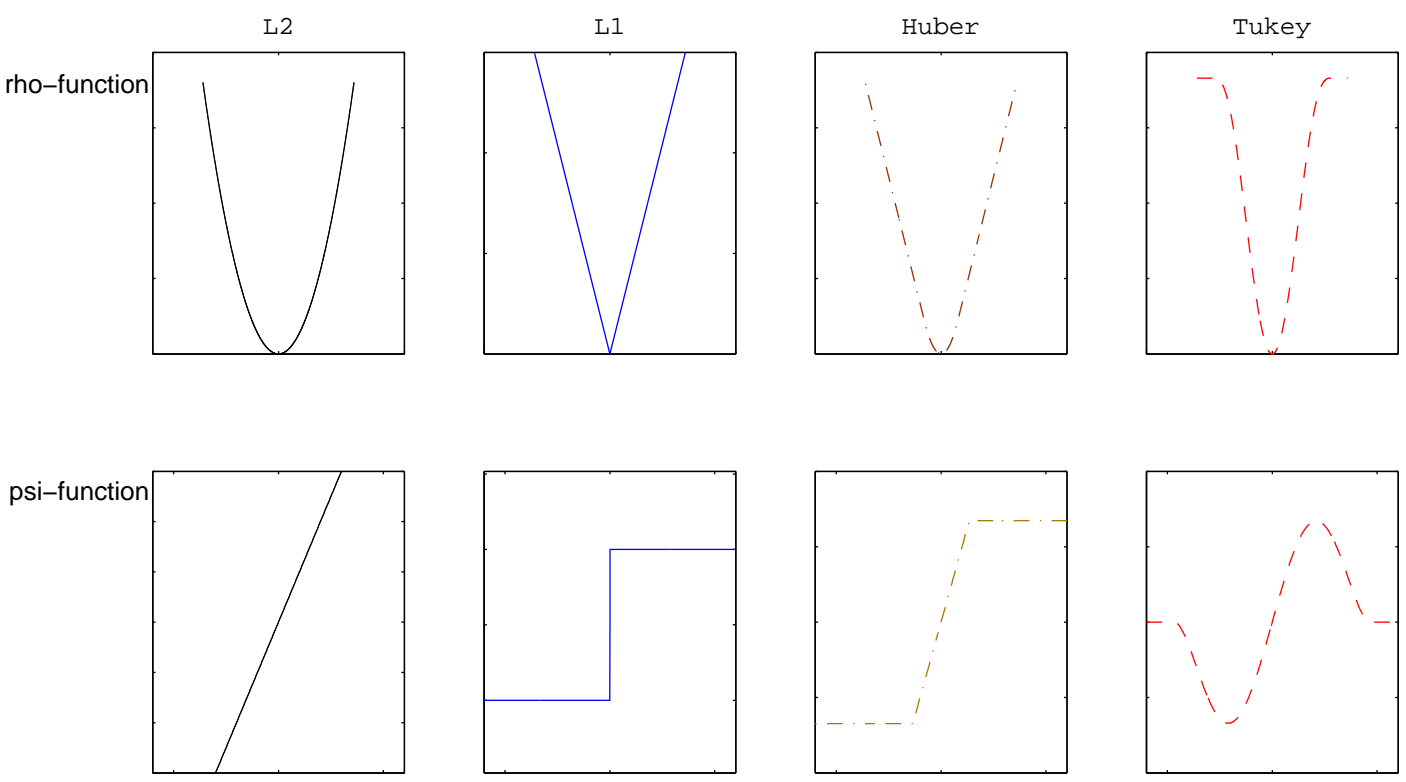

Figure 1: Commonly used loss functions and their corresponding influence functions

where $|\cdot|_{1}$ represents the $L_{1}$ norm, $\rho(\cdot)$ is a robust loss function introduced in Section 3.1, and $\left\{\lambda_{k}, k=1, \cdots, d\right\}$ are the nonnegative regularization parameters.

Noting that $\rho^{\prime}(t)=t \rho^{\prime}(t) / t$, the minimization of (5) can be done using the traditional least-squares-based sparse MAVE in (3) with updated kernel weight

$$
w_{i j}^{*}=w_{i j} W\left(\hat{\varepsilon}_{i j}\right)
$$

where

$$
\begin{gathered}
W\left(\hat{\varepsilon}_{i j}\right)=\frac{\rho^{\prime}\left(\hat{\varepsilon}_{i j}\right)}{\hat{\varepsilon}_{i j}}=\frac{\psi_{c}\left(\hat{\varepsilon}_{i j}\right)}{\hat{\varepsilon}_{i j}}, \\
\hat{\varepsilon}_{i j}=y_{i}-\left\{\hat{a}_{j}+\hat{\mathbf{b}}_{j}^{T} \hat{\mathbf{B}}^{T}\left(\mathbf{x}_{i}-\mathbf{x}_{j}\right)\right\}, \\
w_{i j}=\frac{K_{h}\left\{\hat{\mathbf{B}}^{T}\left(\mathbf{x}_{i}-\mathbf{x}_{j}\right)\right\}}{\sum_{l=1}^{n} K_{h}\left\{\hat{\mathbf{B}}^{T}\left(\mathbf{x}_{l}-\mathbf{x}_{j}\right)\right\}},
\end{gathered}
$$

and $K_{h}(\nu)=h^{-1} K(\nu / h)$ with $K(\nu)$ being a symmetric kernel function and $h$ being the bandwidth. $\left\{\hat{\mathbf{B}},\left(\hat{a}_{j}, \hat{\mathbf{b}}_{j}\right), j=1, \ldots, n\right\}$ are some initial estimator. With the reconstructed 
weight function $w_{i j}^{*}$, the bounded influence function $\psi(\cdot)$ helps put less weights on those observations with large errors and thus achieve robustness. In addition, similar to Č́ížek and Härdle (2006), the original least-squares based algorithm in sparse MAVE can be employed here to minimize the objective function (5) after we replace $w_{i j}$ in (3) by $w_{i j}^{*}$ in (6).

Based on the above discussion, we propose the following estimation algorithm to minimize the objective function (5).

Algorithm 3.1. For a given sample $\left\{\left(y_{i}, \boldsymbol{x}_{i}\right), i=1, \cdots, n\right\}$,

- Step 1: Obtain an initial robust estimator $\left\{\hat{\boldsymbol{B}},\left(\hat{a}_{j}, \hat{\boldsymbol{b}}_{j}\right), j=1, \ldots, n\right\}$ in (4), such as using $\rho_{0}(t)=|t|_{1}$;

- Step 2: Calculate $w_{i j}^{*}$ in (6) from the current estimators;

- Step 3: Replace $w_{i j}$ by $w_{i j}^{*}$ in (3), and update the estimator with the least squares based sparse MAVE algorithm.

1. For given $\hat{\boldsymbol{B}}$, update $\left(a_{j}, \boldsymbol{b}_{j}\right)$ where $j=1, \cdots, n$, from the following quadratic minimization problem

$$
\min _{a_{j}, \boldsymbol{b}_{j}, j=1, \ldots, n}\left(\sum_{i=1}^{n}\left[y_{i}-\left\{a_{j}+\boldsymbol{b}_{j}^{T} \hat{\boldsymbol{B}}^{T}\left(\boldsymbol{x}_{i}-\boldsymbol{x}_{j}\right)\right\}\right]^{2} w_{i j}^{*}\right) .
$$

2. For given $\left(\hat{a}_{j}, \hat{\boldsymbol{b}}_{j}\right), j=1, \cdots, n$, solve $\boldsymbol{B}$ from the following constrained quadratic minimization problem

$$
\min _{\boldsymbol{B}: \boldsymbol{B}^{T} \boldsymbol{B}=I_{d}}\left(\sum_{j=1}^{n} \sum_{i=1}^{n}\left[y_{i}-\left\{\hat{a}_{j}+\hat{\boldsymbol{b}}_{j}^{T} \boldsymbol{B}^{T}\left(\boldsymbol{x}_{i}-\boldsymbol{x}_{j}\right)\right\}\right]^{2} w_{i j}^{*}+\sum_{k=1}^{d} \lambda_{k}\left|\boldsymbol{\beta}_{k}\right|_{1}\right) .
$$

3. Iterate between the previous two steps until convergence in the estimation of $\boldsymbol{B}$.

- Step 4: Iterate between Step 2 and Step 3 until convergence.

Based on our empirical experience, the proposed Algorithm 3.1 usually converges within 5 to 10 iterations. However, one might further speed up the computation based on the 
one-step M-estimation as discussed in Fan and Jiang (1999), Welsch and Ronchetti (2002), and Čížek and Härdle (2006). Therefore, we can just simply run one iteration from Step 1 to Step 3 in Algorithm 3.1.

\subsection{Choice of $c$}

Note that the tuning parameter $c$ in the robust loss function involves the error standard deviation $\sigma$, such as $c=1.345 \sigma$ in Huber function. This $\sigma$ is usually unknown and needs to be estimated. In practice, we can estimate $\sigma$ based on some initial estimate. One robust choice is the median absolute deviation (MAD) as

$$
\hat{\sigma}=\operatorname{Median}\left(\left|\hat{\varepsilon}_{i}-\operatorname{Median}\left(\hat{\varepsilon}_{i}\right)\right|\right) / 0.675 \text {. }
$$

The tuning constant in the value $c$, such as 1.345 for Huber function and 4.685 for Tukey's bisquare function used in our numerical studies, can also be adjusted to reflect the proportion of possible outliers in the data. Essentially, the choice of $c$ is a balance between resistance to outliers and estimation efficiency. More details can be found in Wang et al. (2007) and the references therein.

\subsection{Determination of the dimension d}

The estimation of the structural dimension $d$ is another important task in sufficient dimension reduction. In this section, we propose a robust cross-validation (CV) procedure to determine the optimal dimension $d$. Different from the $L_{1}$-based CV used in Čížek and Härdle (2006), we propose to use a robust CV based on Tukey's bisquare loss function, where the Tukey's bisquare loss function is

$$
\rho(t)= \begin{cases}1-\left[1-(t / c)^{2}\right]^{3} & \text { if }|t| \leq c \\ 1 & \text { if }|t|>c\end{cases}
$$


Once we have an estimated $\hat{\mathbf{B}}$ for a given dimension $k$, we can calculate the corresponding $\mathrm{CV}$ value as

$$
C V_{k}=n^{-1} \sum_{i=1}^{n} \rho\left(y_{i}-\frac{\sum_{j \neq i} y_{j} K_{h}\left\{\hat{\mathbf{B}}^{T}\left(\mathbf{x}_{j}-\mathbf{x}_{i}\right)\right\}}{\sum_{l \neq i} K_{h}\left\{\hat{\mathbf{B}}^{T}\left(\mathbf{x}_{l}-\mathbf{x}_{i}\right)\right\}}\right) .
$$

Then the structural dimension $d$ can be estimated by

$$
\hat{d}=\underset{0 \leq k \leq p}{\operatorname{argmin}} C V_{k}
$$

One might also use some other robust loss functions such as Huber's $\rho$ function

$$
\rho(t)= \begin{cases}t^{2} / 2, & \text { if }|t| \leq c \\ c|t|-c^{2} / 2, & \text { if }|t|>c\end{cases}
$$

in (9). Our empirical studies show that Tukey's bisquare loss usually slightly outperforms the Huber's loss function.

\section{Simulation studies}

In this section, we carried out simulation studies to evaluate the finite sample performance of the proposed robust sparse MAVE (rsMAVE) and to compare it with the traditional refined MAVE (rMAVE, Xia et al., 2002), sparse MAVE (sMAVE, Wang and Yin, 2008), and robust MAVE (rtMAVE, Čížek and Härdle, 2006). For measuring the accuracy of the estimates, we adopted the trace correlation $r$ defined by Ye and Weiss (2003) and Zhu and Zeng (2006). Let $\mathcal{S}(A)$ and $\mathcal{S}(B)$ denote the column space spanned by two $p \times d$ matrices of full column rank. Let $P_{A}=A\left(A^{T} A\right)^{-1} A^{T}$ and $P_{B}=B\left(B^{T} B\right)^{-1} B^{T}$ be the projection matrices onto $\mathcal{S}(A)$ and $\mathcal{S}(B)$ respectively, the trace correlation is defined as $r=\sqrt{\frac{1}{d} \operatorname{tr}\left(P_{A} P_{B}\right)}$. Clearly, $0 \leq r \leq 1$. The larger the $r$ is, the closer $\mathcal{S}(A)$ is to $\mathcal{S}(B)$. To measure the effectiveness of variable selection, we used the true positive rate (TPR), defined as the ratio of the number of predictors correctly identified as active to the number of active predictors, and the false positive rate $(\mathrm{FPR})$, defined as the ratio of the number of predictors falsely identified as active to the number of inactive predictors. Ideally we expect to have the TPR 
close to 1 and the FPR close to 0 simultaneously.

We employed a very efficient Lasso algorithm recently proposed by Friedman, Hastie, and Tibshirani (2010) to solve the $L_{1}$ regularized minimization (8). Cyclical coordinate descent methods were used to calculate the solution path for a large number of $\lambda$ at once. We used the Matlab package "glmnet" in all the simulation studies. More details can be found at http://www-stat.stanford.edu/ tibs/glmnet-matlab/. A BIC criterion was used to select the optimal $\lambda$ 's in the Lasso estimation,

$$
B I C_{\lambda}=n \log \left(\frac{R S S_{\lambda}}{n}\right)+\log (n) p_{\lambda}
$$

where $R S S_{\lambda}$ is the residual sum of squares from the Lasso fit, and $p_{\lambda}$ denotes the number of non-zero coefficients. More details can be found in Wang and Yin (2008). Similar to Čížek and Härdle (2006), the robust CV was used to select the bandwidth $h$ in the kernel estimation.

\subsection{Direction estimation and variable selection}

The data $\left\{\left(\mathbf{x}_{1}, y_{1}\right), \ldots,\left(\mathbf{x}_{n}, y_{n}\right)\right\}$ were generated from the model

$$
y=\frac{\boldsymbol{\beta}_{1}^{T} \mathbf{x}}{0.5+\left(1.5+\boldsymbol{\beta}_{2}^{T} \mathbf{x}\right)^{2}}+\epsilon,
$$

where $\boldsymbol{\beta}_{1}=(1,0, \cdots, 0)^{T}, \boldsymbol{\beta}_{2}=(0,1,0, \cdots, 0)^{T}$, and $\mathbf{x}=\left(x_{1}, \cdots, x_{10}\right)^{T}$ is a 10-dimensional predictor. Therefore, the structural dimension is $d=2$. We considered both independent and correlated cases for $\mathbf{x}:$ (a) $\mathbf{x} \sim N_{10}\left(\mathbf{0}_{10}, \mathbf{I}_{10}\right)$ and (b) $\mathbf{x} \sim N_{10}\left(\mathbf{0}_{10}, \boldsymbol{\Sigma}\right)$, where $(i, j)^{t h}$ element of $\boldsymbol{\Sigma}$ is $0.5^{|i-j|}$. Four error distributions of $\epsilon$ were investigated:

1. $N(0,1)$, the standard normal errors. This density serves as a benchmark with no outliers;

2. $t_{3} / \sqrt{3}$, the scaled $t$-distribution with 3 degree of freedom;

3. $0.95 N(0,1)+0.05 N\left(0,10^{2}\right)$, the standard normal errors contaminated by $5 \%$ normal errors with mean 0 and standard deviation 10; 
4. $0.95 N(0,1)+0.05 U(-50,50)$, the standard normal errors contaminated by $5 \%$ errors from a uniform distribution in between -50 and 50 .

We estimated the EDR directions based on rMAVE (the refined MAVE), sMAVE (the sparse MAVE), rtMAVE (the robust MAVE), and rsMAVE (the robust sparse MAVE). Various sample sizes, $n=100,200$, and 400, were examined and 200 data replicates were drawn in each case. We tried both Huber's loss function and Tukey's bisquare loss function in simulations. Both loss functions gave very similar estimates, although Tukey's bisquare loss gave slightly better results than Huber's loss in some cases. To simplify the presentation, we only reported the results from Tukey's loss. Table 1 and 2 give the summary of comparison among these four different methods for independent and correlated predictors, respectively. The mean and the standard error of the trace correlation $r$ are reported, together with the TPR and FPR for the effectiveness of variable selection.

Table 1: Estimation accuracy for independent predictors

\begin{tabular}{|c|c|c|c|c|c|c|c|}
\hline \multirow[b]{2}{*}{$\epsilon$} & \multirow[b]{2}{*}{$n$} & \multicolumn{4}{|c|}{ The trace correlation $r$} & \multicolumn{2}{|c|}{ TPR \& FPR } \\
\hline & & rMAVE & sMAVE & rtMAVE & rsMAVE & sMAVE & rsMAVE \\
\hline \multirow[t]{3}{*}{1} & 100 & & $0.876(0.144)$ & $0.717(0.103)$ & $0.850(0.160)$ & $(0.853,0.126)$ & $\left(\begin{array}{ll}0.818, & 0.142)\end{array}\right.$ \\
\hline & 200 & $0.880(0.079)$ & $0.970(0.078)$ & $0.845(0.085)$ & $0.958(0.094)$ & $(0.968,0.062)$ & $\left(\begin{array}{ll}0.958, & 0.083)\end{array}\right.$ \\
\hline & 400 & $0.959(0.025)$ & $0.999(0.007)$ & $0.936(0.041)$ & $0.998(0.005)$ & $(1.000,0.037)$ & $(1.000,0.052)$ \\
\hline \multirow[t]{3}{*}{2} & 100 & $0.764(0.110)$ & $0.877(0.151)$ & $0.791(0.097)$ & $0.907(0.129)$ & $(0.873,0.178)$ & $(0.897,0.159)$ \\
\hline & 200 & $0.885(0.089)$ & $0.962(0.089)$ & & & $(0.960,0.092)$ & $\left(\begin{array}{ll}0.998, & 0.093)\end{array}\right.$ \\
\hline & 400 & $0.955(0.047)$ & & & & $(0.995,0.068)$ & $\left(\begin{array}{ll}1.000, & 0.083\end{array}\right)$ \\
\hline \multirow[t]{3}{*}{3} & 100 & $0.599(0.134)$ & $0.659(0.231)$ & $0.706(0.102)$ & $0.862(0.143)$ & $(0.738,0.407)$ & $\left(\begin{array}{ll}0.820, & 0.153\end{array}\right)$ \\
\hline & 200 & $0.623(0.115)$ & $0.685(0.208)$ & & & $(0.708,0.374)$ & $\left(\begin{array}{ll}0.933, & 0.088)\end{array}\right.$ \\
\hline & 400 & $0.697(0.119)$ & $0.737(0.209)$ & & $0.998(0.007)$ & $(0.755,0.343)$ & $(1.000,0.061)$ \\
\hline \multirow[t]{3}{*}{4} & 100 & $0.488(0.139)$ & $0.451(0.262)$ & $0.668(0.106)$ & $0.837(0.154)$ & $(0.683,0.632)$ & $(0.800,0.161)$ \\
\hline & 200 & $0.469(0.128)$ & $0.389(0.278)$ & $0.797(0.093)$ & $0.958(0.097)$ & $(0.593,0.540)$ & $\left(\begin{array}{ll}0.953, & 0.075\end{array}\right)$ \\
\hline & 400 & $0.469(0.130)$ & $0.439(0.279)$ & $0.919(0.057)$ & $0.997(0.021)$ & $(0.573,0.518)$ & $(0.998,0.066)$ \\
\hline
\end{tabular}

From the summary of all four different error distributions, we have the following findings.

1. For the standard normal errors, the robust estimation procedures gave comparable results as the least squares based methods, i.e., rtMAVE performed similar to rMAVE 
Table 2: Estimation accuracy for correlated predictors

\begin{tabular}{|c|c|c|c|c|c|c|c|}
\hline \multirow[b]{2}{*}{$\epsilon$} & \multirow[b]{2}{*}{$n$} & \multicolumn{4}{|c|}{ The trace correlation $r$} & \multicolumn{2}{|c|}{ TPR \& FPR } \\
\hline & & rMAVE & sMAVE & rtMAVE & rsMAVE & sMAVE & rsMAVE \\
\hline \multirow[t]{3}{*}{1} & 100 & $0.668(0.099)$ & $0.805(0.156)$ & $0.659(0.094)$ & $0.802(0.146)$ & $(0.797,0.164)$ & $(0.792,0.198)$ \\
\hline & 200 & $0.790(0.099)$ & $0.917(0.123)$ & $0.762(0.090)$ & $0.887(0.138)$ & $\left(\begin{array}{lll}0.945, & 0.101)\end{array}\right.$ & $\left(\begin{array}{ll}0.902, & 0.126)\end{array}\right.$ \\
\hline & 400 & $0.916(0.061)$ & $0.975(0.074)$ & $0.854(0.077)$ & $0.969(0.083)$ & $(0.998,0.079)$ & $(0.993,0.102)$ \\
\hline \multirow[t]{3}{*}{2} & 100 & $0.709(0.105)$ & $0.828(0.148)$ & $0.722(0.095)$ & $0.847(0.145)$ & $(0.863,0.209)$ & $\left(\begin{array}{ll}0.900, & 0.239\end{array}\right)$ \\
\hline & 200 & $0.797(0.094)$ & $0.906(0.126)$ & $0.836(0.081)$ & $0.949(0.100)$ & $\left(\begin{array}{lll}0.933, & 0.139)\end{array}\right.$ & $\left(\begin{array}{ll}0.985, & 0.153)\end{array}\right.$ \\
\hline & 400 & $0.902(0.083)$ & $0.968(0.082)$ & $0.920(0.056)$ & $0.995(0.030)$ & $(0.990,0.109)$ & $\left(\begin{array}{ll}1.000, & 0.139\end{array}\right)$ \\
\hline \multirow[t]{3}{*}{3} & 100 & $0.559(0.150)$ & $0.646(0.249)$ & $0.648(0.095)$ & $0.780(0.141)$ & $\left(\begin{array}{lll}0.705, & 0.366)\end{array}\right.$ & $\left(\begin{array}{ll}0.767, & 0.203)\end{array}\right.$ \\
\hline & 200 & $0.595(0.116)$ & $0.710(0.169)$ & $0.742(0.091)$ & $0.879(0.135)$ & $(0.708,0.326)$ & $(0.907,0.153)$ \\
\hline & 400 & $0.651(0.107)$ & $0.756(0.183)$ & $0.831(0.080)$ & $0.957(0.097)$ & $(0.797,0.306)$ & $\left(\begin{array}{lll}0.993, & 0.122)\end{array}\right.$ \\
\hline \multirow[t]{3}{*}{4} & 100 & $0.476(0.144)$ & $0.453(0.273)$ & $0.633(0.106)$ & $0.788(0.147)$ & $\left(\begin{array}{ll}0.693, & 0.623)\end{array}\right.$ & $\left(\begin{array}{lll}0.787, & 0.214\end{array}\right)$ \\
\hline & 200 & $0.449(0.131)$ & $0.418(0.292)$ & $0.730(0.086)$ & $0.887(0.131)$ & $(0.570,0.509)$ & $(0.927,0.149)$ \\
\hline & 400 & $0.460(0.129)$ & $0.461(0.281)$ & $0.827(0.086)$ & $0.967(0.082)$ & $(0.630,0.489)$ & $(0.995,0.127)$ \\
\hline
\end{tabular}

and rsMAVE performed similar to sMAVE. In addition, we can see that the sMAVE and rsMAVE achieved better accuracy than rMAVE and rtMAVE respectively due to the sparsity of the model.

2. The MAVE did show some robustness when the errors were from the scaled t-distribution, as mentioned in the original MAVE paper. But with the inclusion of larger outliers in the response as in the error distributions 3 and 4, the least squares based methods failed to estimate the true directions and to select the informative covariates.

3. In the error distributions 2 to 4 , the robust estimation procedures performed almost equally well as they did in the cases without outliers. By selecting the informative covariates, the rsMAVE outperformed the rtMAVE in terms of estimation accuracy and also eased the subsequent model building. In addition, rsMAVE also outperformed sMAVE, especially in the error distributions 3 and 4 where some large outliers appear.

Based on the above observations, we can conclude that the proposed rsMAVE procedure provided very consistent estimates with good direction estimation and variable selection accuracy in all error distributions considered and had overall best performance among all 
four methods considered.

\subsection{Estimation of the structural dimension d}

In this section, we evaluate the finite-sample performance of our proposed robust $\mathrm{CV}$ procedure based on Tukey's bisquare loss function for the estimation of dimension $d$. Data were generated in the same manner as in model (10). Therefore, the true value of $d$ is 2 . Here we report only the results from the independent predictors with sample size $n=100$ and 200. For each case, 200 data replicates were used. Table 3 summarizes the frequency of estimated $d$ out of 200 data replicates. For comparison, the results from $L_{1}$-based $\mathrm{CV}$ were also reported. We can see that the proposed robust CV procedure provided very consistent estimation for different error distributions. The method performed reasonably well for the cases with outliers, although slightly worse than those without outliers. The robust CV based on Tukey's bisquare function slightly outperforms the $L_{1}$-based CV for distribution 3 and 4, where extreme outliers occur. This can be explained from the influence function where the bisquare function suppresses all the extreme outliers, while the $L_{1}$ puts less weights on them.

Table 3: Frequency of estimated $d$ out of 200 data replicates

\begin{tabular}{cccccccccccc}
\hline \multicolumn{1}{c}{$C V_{\text {Tukey }}$} & \multicolumn{7}{c}{$C V_{L_{1}}$} \\
\hline 1 & $n$ & $d=1$ & $d=2$ & $d=3$ & $d=4$ & $d \geq 5$ & $d=1$ & $d=2$ & $d=3$ & $d=4$ & $d \geq 5$ \\
\hline & 100 & 9 & $\mathbf{1 5 5}$ & 35 & 1 & 0 & 7 & $\mathbf{1 5 3}$ & 40 & 0 & 0 \\
& 200 & 2 & $\mathbf{1 7 8}$ & 20 & 0 & 0 & 4 & $\mathbf{1 8 0}$ & 15 & 1 & 0 \\
\hline 2 & 100 & 12 & $\mathbf{1 4 6}$ & 40 & 2 & 0 & 9 & $\mathbf{1 4 1}$ & 47 & 3 & 0 \\
& 200 & 3 & $\mathbf{1 7 2}$ & 24 & 1 & 0 & 5 & $\mathbf{1 7 6}$ & 19 & 0 & 0 \\
\hline 3 & 100 & 31 & $\mathbf{1 0 0}$ & 47 & 15 & 7 & 46 & $\mathbf{9 1}$ & 43 & 17 & 3 \\
& 200 & 5 & $\mathbf{1 3 2}$ & 52 & 10 & 1 & 16 & $\mathbf{1 2 0}$ & 51 & 10 & 3 \\
\hline 4 & 100 & 43 & $\mathbf{9 5}$ & 32 & 12 & 18 & 49 & $\mathbf{9 0}$ & 46 & 10 & 5 \\
& 200 & 16 & $\mathbf{1 3 4}$ & 26 & 8 & 16 & 21 & $\mathbf{1 2 2}$ & 32 & 19 & 6 \\
\hline
\end{tabular}




\section{Logo design data}

Wang and Yin (2008) studied a logo design data collected by Henderson and Cote (1998). The objective is to understand how logo design characteristics may influence consumers' reactions to logos. There are 195 observations and 22 predictors in the data, and the response variable $y$ is the logo effect, which ranges from -2.55 to 2.16 with variance around 1. Sparse MAVE (sMAVE; Wang and Yin, 2008) identified 1 significant direction with 9 informative variables out of the 22 as listed in Table 4.

To verify our robust variable selection procedure, we re-analyzed this data set by including some outliers in the response variable. Two cases were considered in the analysis, a single outlier and 5\% contaminated observations. For each case, the outliers were randomly generated by increasing the value $y_{i}$ to $y_{i}+c$ and the results from $c=10$ and 20 were reported. From our numerical experience, the pattern were very consistent over different repetitions. In Table 4, we compared the variable selection performance of sMAVE and rsMAVE. To evaluate the estimation accuracy, the correlation between each estimated direction and the directions from sMAVE without outliers, denoted by $\operatorname{corr}\left(\hat{\beta}, \hat{\beta}_{s 0}\right)$, was also presented.

Table 4: Comparison of variable selection. The last column reports the correlations between each estimated directions and the directions from sMAVE without outliers.

Selected variables $\quad \operatorname{corr}\left(\hat{\beta}, \hat{\beta}_{s 0}\right)$

\begin{tabular}{ccccc} 
Outliers & sMAVE & rsMAVE & sMAVE & rsMAVE \\
\hline No outlier & $(2,3,4,8,9,10,12,14,17)$ & $(2,3,6,8,9,10,12,14,17)$ & 1 & 0.9896 \\
Single outlier $(c=10)$ & $(1,3,8,9,10,11,12,14,17,21)$ & $(2,3,8,9,10,12,14,17)$ & 0.9132 & 0.9893 \\
Single outlier $(c=20)$ & $(1,3,8,9,10,11,12,14,21)$ & $(2,3,8,9,10,12,14,17)$ & 0.8251 & 0.9892 \\
$5 \%$ outliers $(c=10)$ & all variables except $(3,10,16,20)$ & $(2,3,8,9,10,12,14,17)$ & 0.3346 & 0.9904 \\
$5 \%$ outliers $(c=20)$ & all variables except $(16,20)$ & $(2,3,8,9,10,12,14,17)$ & 0.0276 & 0.9883 \\
\hline
\end{tabular}

From the summary, we can see that the performance of sMAVE and rsMAVE are very similar for the original data. After adding outliers, sMAVE is clearly affected in both direction estimation and variable selection. But rsMAVE gives very consistent results, even with $5 \%$ extreme values. 


\section{Conclusion}

In this article, we proposed a robust model-free variable selection method, rsMAVE, which combines the strength of both robust and shrinkage estimation. Our numerical studies demonstrate that the proposed method has better performance than the traditional refined MAVE (rMAVE), the sparse MAVE (sMAVE), and the robust MAVE (rtMAVE) when the model is sparse and outliers exist in the response variables. In addition, a robust cross-validation criterion based on Tukey's bisquare loss function was proposed to select the structural dimension $d$.

We believe that this robust variable selection idea can also be extended to models where the response takes discrete values, such as in logistic regression and Poisson regression. The investigation for such a general class is under way.

\section{Acknowledgments}

The authors are grateful to Professors P.A. Naik and C.L. Tsai for providing us the Logo design data. The authors would like to thank the Editor, an Associate Editor, and three anonymous referees for their valuable comments and suggestions, which led to substantial improvements in the manuscript.

\section{References}

Bellman, R. E. (1961). Adaptive Control Processes. Princeton University Press, Princeton, NJ.

Bondell, H.D. and Li, L. (2008). Shrinkage inverse regression estimation for model free variable selection. Journal of Royal Statistical Society B, 71, 287-299.

Breiman, L. (1995). Better subset regression using the nonnegative Garrote. Technometrics, 37, 373-384.

Breiman, L. (1996). Heuristics of instability and stabilization in model selection. Annals of Statistics, 24, $2350-2383$.

Čížek, P. and Härdle, W. (2006). Robust estimation of dimension reduction space. Computational Statistics and Data Analysis, 51, 545-555.

Cook, R. D. (1998). Regression Graphics: Ideas for studying regressions through graphics. Wiley, New York.

Cook, R. D. (2004). Testing predictor contributions in sufficient dimension reduction. Annals of Statistics, 32, 1062-1092. 
Efron, B., Hasti, T., Johnstone, I. and Tibshirani, R. (2004). Least angle regression. Annals of Statistics, 32, 407-499.

Fan, J. and Jiang, J., (1999). Variable bandwidth and one-step local M-estimator. Science China Series A, $43,65-81$.

Fan, J. and Li, R. (2001). Variable selection via nonconcave penalized likelihood and its oracle properties. Journal of the American Statistical Association, 96, 1348-1360.

Fan, J. and Li, R. (2006). Statistical challenges with high dimensionality: feature selection in knowledge discovery. Proceedings of the international congress of mathematicians, Spain, 2006.

Friedman. J., Hastie, T. and Tibshirani, R. (2010). Regularization paths for generalized linear models via coordinate descent, Journal of Statistical Software, 33, 1-22.

Hampel, F. R., Ronchetti, E. M., Rousseeuw, P. J. and Stahel, W. A. (1986). Robust Statistics: The Approach Based on Influence Functions, Wiley, New York.

Henderson, P. W. and Cote, J. A. (1998). Guidelines for selecting or modifying logos. Journal of Marketing, 62(April), 14-30.

Huber, P. J. (1981). Robust Statistics. Wiley, New York.

Li, K. C. (1991). Sliced inverse regression for dimension reduction (with discussion). Journal of the American Statistical Association, 86, 316-342.

Li, L. (2007). Sparse sufficient dimension reduction. Biometrika, 94, 603-613.

Li, L., Cook, R. D. and Nachtsheim, C. J. (2005). Model-free variable selection. Journal of Royal Statistical Society B, 67, 285-299.

Li, L. and Nachtsheim, C. J. (2006). Sparse sliced inverse regression, Technometrics, 48, 503-510.

Maronna, R. A., Martin, R. D. and Yohai, V. J. (2006). Robust Statistics: Theory and Methods. Wiley, New York.

Ni, L., Cook, R. D. and Tsai, C. L. (2005). A note on shrinkage sliced inverse regression. Biometrika, 92, 242-247.

Rousseeuw, P. J. and Leroy, A. M. (2003). Robust regression and outlier detection, Wiley, New York.

Tibshirani, R. (1996). Regression shrinkage and selection via the lasso. Journal of Royal Statistical Society $B, 58,267-288$.

Wang, Q. and Yin, X. (2008). A nonlinear multi-dimensional variable selection method for high dimensional data: Sparse MAVE. Computational Statistics and Data Analysis, 52, 4512-4520.

Wang, Y., Lin, X., Zhu, M. and Bai, Z. (2007). Robust estimation using the Huber function with a datadependent tuning constant. Journal of Computational and Graphical Statistics, 16, 468-481.

Welsh, A.H. and Ronchetti, E. (2002). A journey in single steps: robust one-step M- estimation in linear regression. Journal of Statistical Planning and Inference, 103, 287-310. 
Xia, Y., Tong, H., Li, W. and Zhu, L. (2002). An adaptive estimation of dimension reduction space. Journal of Royal Statistical Society B, 64, 363-410.

Ye, Z. and Weiss, R. E. (2003). Using the bootstrap to select one of a new class of dimension reduction methods. Journal of the American Statistical Association, 98, 968-979.

Zhou, J. and He, X. (2008). Dimension reduction based on constrained canonical correlation and variable filtering. Annals of Statistics, 36, 1649 - 1668.

Zhu, Y. and Zeng, P. (2006). Fourier methods for estimating the central subspace and the central mean subspace in regression. Journal of the American Statistical Association, 101, 1638-1651.

Zou, H. and Hastie, T. (2005). Regularization and variable selection via the elastic net. Journal of Royal Statistical Society B, 67, 301-320. 